\title{
Investigation of Spring Irrigation and Tillage to Control Overwintering Chilo suppressalis (Walker)
}

\author{
Shuijin Huang ${ }^{1}$, Guanghua Luo ${ }^{2}$, Wenjing Qin ${ }^{1}$, Cheng Gan ${ }^{3}$, Guoquan Wu ${ }^{4}$, Rongzai Xu ${ }^{4}$, Lianghui \\ Lei $^{4}$, Lu Zhang ${ }^{4}$, Zhaojun Han ${ }^{5}$, Yang Sun ${ }^{1, *}$ \\ ${ }^{1}$ Jiangxi Academy of Agricultural Sciences, Nanchang, China \\ ${ }^{2}$ Jiangsu Academy of Agricultural Sciences, Nanjing, China \\ ${ }^{3}$ Plant Protection Station, Jiujiang, China \\ ${ }^{4}$ Agricultural Technology Extension Center, Fengcheng, China \\ ${ }^{5}$ College of Plant Protection, Nanjing Agricultural University, Nanjing, China
}

Email address:

sjhuang@aliyun.com (Shuijin Huang), luogh_cn@163.com (Guanghua Luo),476815535@qq.com(Wenjing Qin), yxxzbz@163.com (Cheng Gan),834956018@qq.com (GuoquanWu),914599581@qq.com (Rongzai Xu), jxsycsfcs@sina.com (Lianghui Lei),435329201@qq.com(Lu Zhang), zjhan@njau.edu.cn(Zhaojun Han), sun2007yang@126.com (Yang Sun)

*Corresponding author

\section{To cite this article:}

Shuijin Huang, Guanghua Luo, Wenjing Qin, Cheng Gan, Guoquan Wu, Rongzai Xu, Lianghui Lei, Lu Zhang, Zhaojun Han, Yang Sun. Investigation of Spring Irrigation and Tillage to Control Overwintering Chilo suppressalis (Walker). International Journal of Applied Agricultural Sciences. Vol. 4, No. 5, 2018, pp. 110-116. doi: 10.11648/j.ijaas.20180405.12

Received: October 4, 2018; Accepted: October 30, 2018; Published: November 29, 2018

\begin{abstract}
Overwintering larvae and pupae are the most important source of first generation of Chilo suppressalis (Walker) in early spring. Aging larvae and pupae harbor strong stress-tolerance and also potential pesticide resistance. Therefore, at present, irrigation and tillage are mainly used for killing the first generation of $C$. suppressalis. However, the efficiency of this method was not clear. To study the exact efficiency, overwintering larvae and pupae were collected from rice straw in field. And they were placed in water to test their tolerance to immersion indoor. The control effect of irrigation and tillage was tested in field and repeated in different years. As a result, in tolerance experiment, the mortality of aging larvae was less than $50 \%$ on day 8 and reached $80 \%$ on day 12 . The mortality of pupae reached $75 \%$ within 72 hours. In the field, the escape rate of larvae was the highest on day $1(4.49 \%)$, after which time it gradually decreased. The cumulative escape rate was $7.89 \%$ within 4 days. When the fields were tilled immediately after immersion, the cumulative escape rate decreased to $1.05 \%$. There was no significant difference in escape rate with different tillage depths. This is the first study to systematically investigate the control effect of irrigation and tillage on overwintering C. suppressalis. And the results showed that aging larvae of $C$. suppressalis had strong tolerance to immersion. But the tolerance of pupa to water was relatively weak. Tillage could effectively reduce the escape rate of aging larvae. So, "Tilling immediately after irrigation" was recommended in early spring. This will provide guidance for agricultural control of $C$. suppressalis.
\end{abstract}

Keywords: Chilo suppressalis, Early Spring Source, Agricultural Control, Irrigation, Tillage

\section{Introduction}

The rice stem borer, Chilo suppressalis (Walker) (Lepidoptera: Crambidae) is one of the most important economic pests in rice and occurs in most Chinese rice-growing areas $[1,2]$. Chemical control is often the first and perhaps the only choice out of many control methods for
C. suppressalis because of its quick response, long duration of efficacy and good control effect. As a result, there are ongoing problems with pesticide resistance and pesticide residues caused by overuse of chemical pesticides [2]. This is contrary to the concept of integrated pest management (IPM), namely, promoting coordination between chemical control and non-chemical control [3]. 
Agricultural control is an important component of non-chemical control. The current agricultural control methods for $C$. suppressalis include: removal of rice straw and stubble from paddy fields, field burning, winter tillage, and spring irrigation [4-7]. Artificial harvesting and mechanical harvesting are the two primary methods used for straw removal. Rice fields that are irregular in size require artificial harvesting. The process of artificial harvesting is usually inefficient because it is limited by human physical attributes. Thus, the amount and height of stubble varies greatly. Although harvest efficiency is high, the stubble height retained is related to rice varieties during mechanical harvest. Moreover, about $80 \%$ of the remaining C. suppressalis are concentrated within $20 \mathrm{~cm}$ from the ground in conventional rice stubble. When planting tall rice species with up to $1.5-\mathrm{m}$ plant height, about $60 \%$ of the remaining $C$. suppressalis are concentrated more than $20 \mathrm{~cm}$ from the ground [8]. The comprehensive consideration of rice varieties, straw and stubble height is needed for mechanical harvest. Burning the fields (straw) after harvest might be the most easiest and effective control method; Jiang et al. (2011) found that burning killed $84.5 \%$ of the remaining insects including $C$. suppressalis. But burning not only pollutes the environment, but also has a huge impact on natural enemies. For winter tillage, there is neither technical complexity nor disadvantages in terms of adverse environmental impacts. However, it gave low (less than 30\%) reduction in borer populations in fields with stubble height of $10 \mathrm{~cm}$ [9].

Irrigation is a traditional agricultural control method. It was tried and practiced as early as 1971 and was found to be simple and effective [10]. Since then, there have been many different combinations of irrigation and other measures. For example, some raised ducks in fallow rice fields with deep water to protect against borers [6]. In this study, the effect of irrigation and the combination of spring tillage and spring irrigation were studied. The aim is to explore the theory and provide evidence for economic, efficient and precise agricultural control of $C$. suppressalis.

\section{Material and Methods}

\subsection{Tolerance of Overwintering Larvae to Immersion}

Overwintering larvae (or $6^{\text {th }}$ instar larvae) of C. suppressalis were collected from the rice straw. The larvae with same size and developmental status were selected for the experiment. About 100 larvae were put into one sealed glass bottle, which was filled with water without bubbles and fixed at the bottom of a plastic bucket. The bucket was filled up with water to about $80 \mathrm{~cm}$ height. After that, the glass bottles were removed from the bucket carefully and the larvae were taken out of the bottle daily. The larval surface was dried on tissue paper for 24 $\mathrm{h}$ and then they were monitored for being live or dead. The mortality rate of larvae was counted after checking. Three bottles were taken daily until the mortality reached $100 \%$. The experiments were performed in triplicate.

\subsection{Tolerance of Pupae to Immersion}

The overwintering larvae of $C$. suppressalis were reared at room temperature after collected from the rice straw. When they became pupae, 60-70 pupae were placed in a gauze bag. The gauze bag was immersed in water and the pupae were kept just under water. The pupae were removed from the water after immersion for several hours. The eclosion rate and mortality rate of pupae was calculated. The pupae were immersed for $0,6,12,24,48,72,96,120$, and $144 \mathrm{~h}$. The experiments were performed in triplicate.

The pupae of indoor susceptible (S) strain C. suppressalis were also treated as above. The susceptible (S) strain of $C$. suppressalis was provided by Dr. Lanzhi Han (Institute of Plant Protection, Chinese Academy of Agricultural Sciences). This strain had been reared on an artificial laboratory diet in a laboratory for over 40 generations without exposure to any insecticides.

\subsection{Irrigation to Control C. suppressalis}

Before irrigation, the insect density of the overwintering larval population in winter fallow fields were investigated. For easy manipulation, large plots larger than $667 \mathrm{~m}^{2}$ and five-point sampling methods were employed. For each point, 25 hills of rice stubble were sampled by the parallel line sampling method and then dissected for borers. The average number of borers in each point were calculated and it represents the density of insects in every plot. The plots of which the density showing no difference by statistical analysis were selected for experiment. A gauze net was placed around the plot and then the plot was filled with water to the top of the remaining straw. The number of larvae on the gauze was counted daily and the larvae were removed. The larvae remaining in the rice straw were sampled from the field. The method was described as above, that is, five-point method and 25 hills of rice stubble were dissected for borers. The effect of limewater for control of $C$. suppressalis was tested by using limewater (calcium oxide: $\mathrm{CaO}$ ) for irrigation. The amount of $\mathrm{CaO}$ applied was $75 \mathrm{~kg}$ per $667 \mathrm{~m}^{2}$ [11].

\subsection{Rotary Tillage Immediately After Irrigation to Control C. suppressalis}

The method of estimating borer density and selecting experimental plots was described as 2.3 Irrigation to control $C$. suppressalis. Rice plots in which there was high proportion of larvae (The proportion of larvae was more than 95\%) were selected to test whether rotary tillage immediately after irrigation affect $C$. suppressalis controlling. The plots were tilled immediately by tractor after irrigation; a gauze net was then placed around the fields. Larvae that escaped on the gauze were caught and enumerated daily. To study whether different tillage depth had an influence on the escape rate of larvae, two different rotary tillers were selected: 1) a small self-propelled diesel rotary cultivator (Model: ChangYu-151, Shandong, China) with a depth of $20 \mathrm{~cm}$, and 2) a large tractor rotary cultivator (Model: IGQQN-250J, Hubei, China) with a depth of $35 \mathrm{~cm}$. 
After tilling, the tilled soil layer and all rice stubble residues were inspected in a $2 \mathrm{~m}^{2}$ sample area at each point (equivalent to about 25 rice hills). The density of live borers per hill was calculated by 20 sampling points. The experiment was conducted in 2017 (from April 1 to 5) and 2018 (from March 26 to 30$)$, respectively.

\subsection{Statistical Analysis}

All experimental treatments (both laboratory and field experiments and including control treatments) were conducted with three replicates. Data were analyzed by ANOVA and followed by Duncan's test using IBM SPSS Statistics for Windows 20.0 (IBM Corp., Armonk, NY, USA). The level of significance was set at $\mathrm{P}<0.05$.

\section{Results}

\subsection{Tolerance of Overwintering Larvae to Immersion}

The overwintering $C$. suppressalis larvae (ageing larvae, or $6^{\text {th }}$ instar larvae) were immersed in an average depth of 70-80 $\mathrm{cm}$. The time of immersion lasted until all the larvae died (Figure 1). Within 8 days of continuous immersion, the mortality of larvae was near but below $50 \%$, and the average mortality rate reached $80 \%$ on day 12 . All larvae were dead by day 17. The results showed that the overwintering larvae harbored incredible tolerance to immersion.

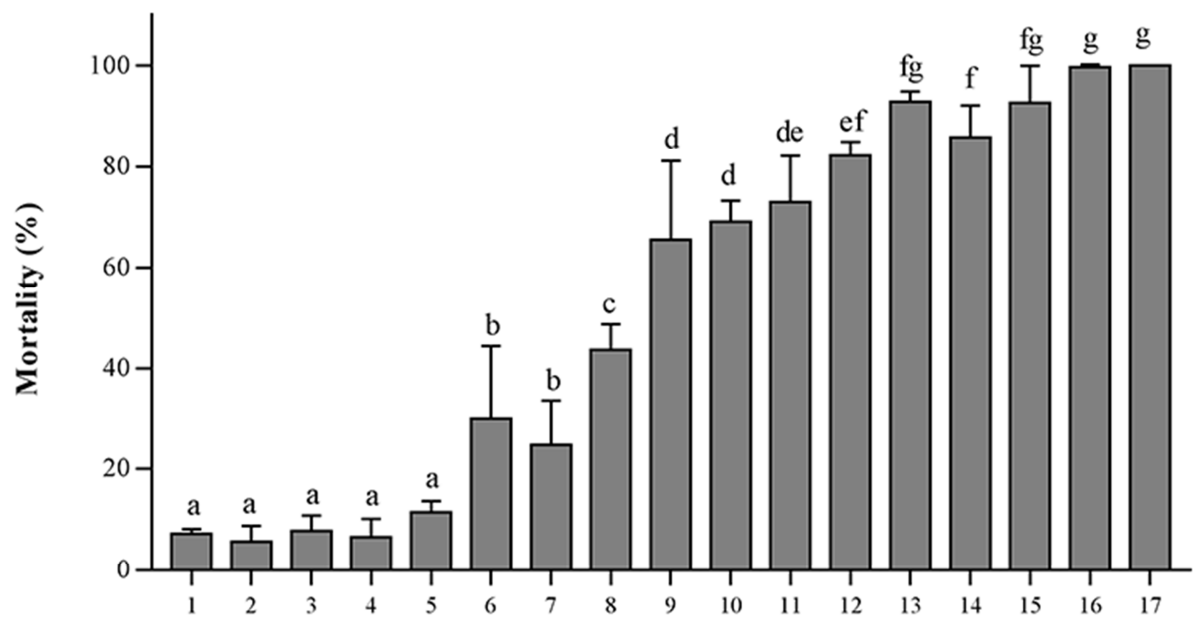

Time of continuously immersed (day)

Figure 1. Mortality of field overwintering larvae with continuous immersion. Values are shown as mean \pm SD (error bars) of three replicates. Multiple comparisons were conducted using Duncan's test with $P<0.05$. Different letters indicate significant differences among means.

\subsection{Tolerance of Pupae to Immersion}

\subsubsection{Tolerance of Overwintering Pupae to Immersion}

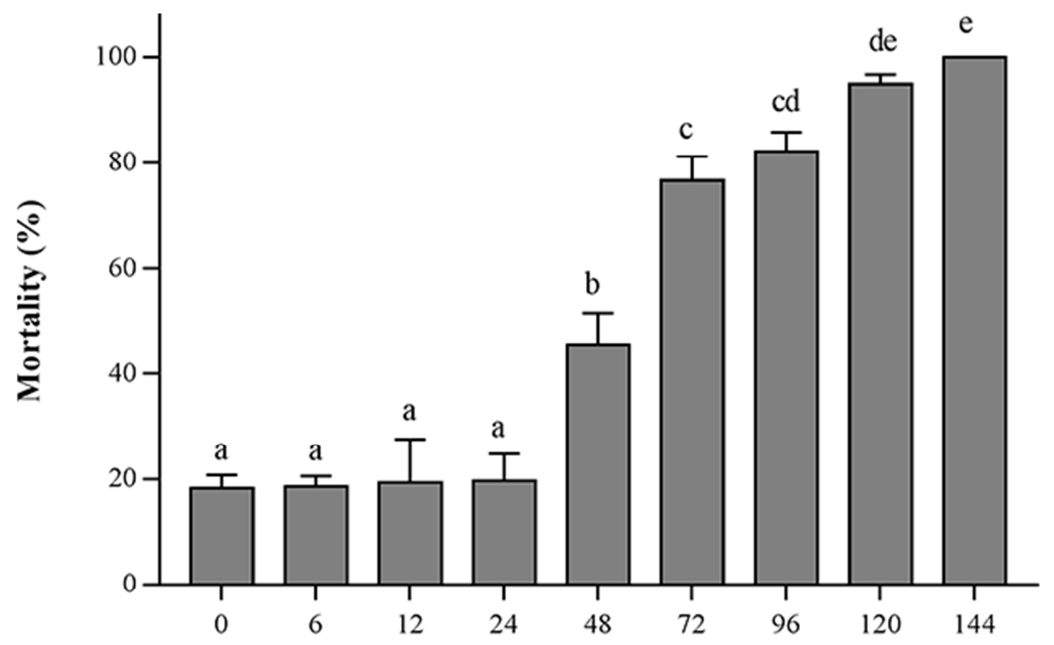

Time of continuously immersed (hour)

Figure 2. Mortality of field overwintering pupae with continuous immersion. Values are shown as mean \pm SD (error bars) of three replicates. Multiple comparisons were conducted using Duncan's test with $P<0.05$. 


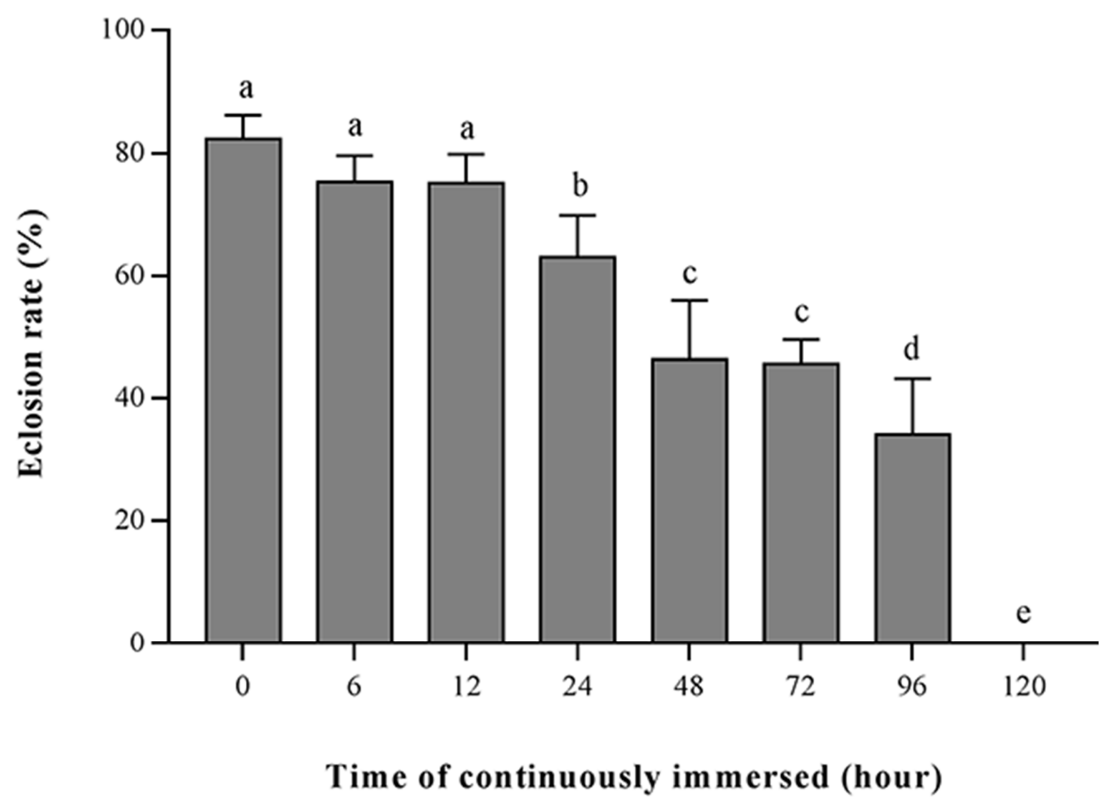

Figure 3. Eclosion rate of field overwintering pupae with continuous immersion. Values are shown as mean \pm SD (error bars) of three replicates. Multiple comparisons were conducted using Duncan's test with $P<0.05$. When the pupae were immersed for 120 h, no pupae could become moths, i.e., eclosion $=0$.

Unlike larvae immersed in deep water, the immersed pupa is only immersed in $5 \mathrm{~cm}$ deep water. The mortality was less than $20 \%$ within $24 \mathrm{~h}$ (Figure 2), increasing to $48 \%$ after $48 \mathrm{~h}$ and to $80 \%$ in the period from 72 to $96 \mathrm{~h}$. All pupae were dead at $144 \mathrm{~h}$. After immersion, the pupae were removed from water to check the eclosion rate of the corresponding time (Figure 3). After immersion for $12 \mathrm{~h}$, the eclosion rate was around $80 \%$, but it declined to $60 \%$ at $24 \mathrm{~h}$. After $48 \mathrm{~h}$ ( 2 days), it dropped to $<50 \%$. No pupae emerged after immersion for $120 \mathrm{~h}$.

\subsubsection{Tolerance of Indoor Pupae to Immersion and Comparison to Overwintering Pupae}

Under the same conditions, the pupae of the indoor S strain were compared with the overwintering pupae for immersion tolerance. When compared with the overwintering pupae in the field (Table 1), the mortality of S strain pupae immersed for $0 \mathrm{~h}$ was significantly lower than that of overwintering pupae $(6.67 \%$ vs $18.25 \%$, $\mathrm{P}$ value $<0.05)$. However, the average mortality of the two strains at different immersion times was similar. The emergence rates of remaining $\mathrm{S}$ strain live pupae were significantly higher than those of the field strain at 6, 24, 48, 72 and $96 \mathrm{~h}$ (Table 2). The emergence rates of both strains were $>70 \%$ after immersion in water for $12 \mathrm{~h}$. The emergence rate of the S strain was still $\sim 70 \%$ at $72 \mathrm{~h}$, while the emergence rate of the field strain was $<50 \%$ after 48 h.

Table 1. Comparison of pupal mortality between indoor $S$ strain and field strain.

\begin{tabular}{|c|c|c|c|}
\hline \multirow{2}{*}{ Time of continuous immersion (h) } & \multicolumn{2}{|l|}{ Mean \pm SD $(\%)$} & \multirow{2}{*}{ Significance analysis ( $P$ value) } \\
\hline & Indoor S strain & Field strain & \\
\hline 0 & $6.67 \pm 1.67$ & $18.25 \pm 2.50$ & 0.002616017 \\
\hline 6 & $13.97 \pm 2.69$ & $18.57 \pm 1.96$ & 0.074877187 \\
\hline 12 & $7.12 \pm 5.07$ & $19.37 \pm 8.18$ & 0.092161625 \\
\hline 24 & $16.58 \pm 2.96$ & $19.63 \pm 5.13$ & 0.423399698 \\
\hline 48 & $35.65 \pm 6.00$ & $45.38 \pm 5.98$ & 0.117348168 \\
\hline 72 & $76.22 \pm 3.65$ & $76.60 \pm 4.59$ & 0.915521927 \\
\hline 120 & $96.67 \pm 1.67$ & $94.85 \pm 1.80$ & 0.267652752 \\
\hline 144 & 100.00 & 100.00 & 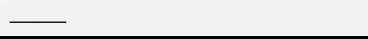 \\
\hline
\end{tabular}

Table 2. Comparison of eclosion rate between indoor $S$ strain and field strain.

\begin{tabular}{llll}
\hline \multirow{2}{*}{ Time of continuous immersion $(\mathbf{h})$} & \multicolumn{2}{l}{ Mean \pm SD (\%) } & Significance analysis (P value) \\
\cline { 2 - 4 } & Indoor S strain & Field strain & 0.234418336 \\
0 & $88.17 \pm 6.07$ & $75.22 \pm 4.37$ & 0.018919048 \\
6 & $86.83 \pm 3.09$ & $75.05 \pm 4.87$ & 0.089971025 \\
12 & $82.60 \pm 3.04$ & $63.07 \pm 6.73$ & 0.048675322 \\
24 & $80.26 \pm 6.29$ & $46.26 \pm 9.76$ & 0.003864280 \\
48 & $77.97 \pm 2.22$ & $45.55 \pm 3.96$ & 0.001510500 \\
72 & $69.58 \pm 3.41$ & $34.16 \pm 9.07$ & 0.012483800 \\
96 & $64.55 \pm 8.15$ & 0 & - \\
\hline
\end{tabular}




\subsection{Influence of Immersion Time on the Escape Rate of Overwintering Larvae}

Three plots of winter fallow field with similar abundance of larvae were selected for the immersion experiment. The method of estimating borer density was described as 2.3 Irrigation to control C. suppressalis. A large proportion of the escaped larvae were found crawling on the gauze, while a small proportion of the remaining larvae were still hiding in the rice straw. The escaped larvae were collected to calculate the escape rate and the larvae hiding in the rice straw were collected to calculate the residual rate daily until day 4 (Table $3)$. The escape rate declined from day $1(4.49 \%)$ to day 3 $(0.52 \%)$ and continued to decline to day $4(0.07 \%)$, although the decline from day 3 to 4 was not statistically significant. The residual rate remained high for the first 3 days within the range of $62.95 \%$ to $44.15 \%$; it declined to $17.46 \%$ at day 4 . The cumulative escape rate after 4 days was $7.89 \%$.

Table 3. Influence of immersion time on escape rate and residual rate of overwintering C. suppressalis.

\begin{tabular}{|c|c|c|c|c|}
\hline \multirow{2}{*}{ Time of continuous immersion (days) } & \multicolumn{2}{|l|}{ Escape rate } & \multicolumn{2}{|l|}{ Residual rate } \\
\hline & Mean \pm SD $(\%)$ & Multiple comparison * & Mean \pm SD $(\%)$ & Multiple comparison \\
\hline 1 & $4.49 \pm 1.32$ & $a * *$ & $62.95 \pm 13.90$ & $a * *$ \\
\hline 2 & $2.82 \pm 0.71$ & $\mathrm{~b}$ & $53.74 \pm 7.20$ & a \\
\hline 3 & $0.52 \pm 0.20$ & $\mathrm{c}$ & $44.15 \pm 14.10$ & $\mathrm{a}$ \\
\hline 4 & $0.07 \pm 0.03$ & $\mathrm{c}$ & $17.46 \pm 8.00$ & $\mathrm{~b}$ \\
\hline Cumulative & $7.89 \pm 1.76$ & - & - & - \\
\hline
\end{tabular}

* Different letters indicate statistical difference at $\mathrm{P}<0.05$

** The sum of escape rate and residual rate of day 1 in Table 3 is not $100 \%$. It may be caused by statistical methods. Or some escaped borers were probably eaten by the birds. Because a lot of birds were found flying above the plots every day when the data was recorded.

However, it was interesting that the sum of escape rate and residual rate of day 1 in Table 3 is not $100 \%$. It may be caused by statistical methods. But some escaped borers were probably eaten by the birds. Because a lot of birds were found flying above the plots every day when the data was recorded.

\subsection{Influence of Limewater Immersion on the Escape Rate of Overwintering Larvae}

Acid soil is widely distributed in the Jiangxi area. It is common practice in this region to neutralize acid soil by mixing with $\mathrm{CaO}\left(75 \mathrm{~kg}\right.$ per $\left.667 \mathrm{~m}^{2}\right)$. Another three fields were selected, similar to those described in section 3.3. $\mathrm{CaO}$ was added into the water during the immersion process. There were no statistical significant differences between immersion with or without $\mathrm{CaO}$ regardless of immersion time (Table 4). However, the escape rate of the limewater treatment was lower than that of the water treatment for days 1 to 3 and the residual rate was higher in the limewater treatment for all time periods.

Table 4. Influence of limewater on escape rate and residual rate of overwintering C. suppressalis.

\begin{tabular}{|c|c|c|c|}
\hline \multirow{2}{*}{ Time of continuous immersion (days) } & \multirow{2}{*}{ Treatment * } & \multicolumn{2}{|c|}{ Mean \pm SD (\%) } \\
\hline & & Escape rate & Residual rate \\
\hline \multirow{2}{*}{1} & water & $4.49 \pm 1.32$ & $62.95 \pm 13.90$ \\
\hline & limewater & $3.83 \pm 1.14$ & $72.48 \pm 8.42$ \\
\hline \multirow{2}{*}{2} & water & $2.82 \pm 0.71$ & $53.74 \pm 7.20$ \\
\hline & limewater & $1.08 \pm 1.37$ & $60.77 \pm 10.72$ \\
\hline \multirow{2}{*}{3} & water & $0.52 \pm 0.20$ & $44.15 \pm 14.10$ \\
\hline & limewater & $0.47 \pm 0.12$ & $52.30 \pm 22.37$ \\
\hline \multirow{2}{*}{4} & water & $0.07 \pm 0.03$ & $17.46 \pm 8.00$ \\
\hline & limewater & $0.13 \pm 0.09$ & $17.52 \pm 8.08$ \\
\hline
\end{tabular}

* There were no significant differences between water and limewater for any treatment.

\subsection{Tillage to Control Overwintering Larvae of C. suppressalis}

Tillage is one of the most common farming operations. The effect of tillage immediately after immersion on control of overwintering larvae was tested in the field. The escape rate after tillage was $0.68 \%, 0.21 \%$ and $0.16 \%$ on days 1,2 and 3 , respectively; no escaped $C$. suppressalis was found on day 4 (Table 5). The cumulative escape rate over 4 days was $1.06 \%$, which was lower than that of day $1(4.49 \%)$ and day $2(2.82 \%)$ without tillage (Table 3), and significantly lower than the cumulative escape rate without tillage over 4 days $(7.89 \%$; Table 3).
Table 5. Effect of tillage after immersion for different time on escape rate.

\begin{tabular}{lll}
\hline Days after tillage & $\begin{array}{l}\text { Escape rate (mean } \pm \\
\text { SD (\%)) }\end{array}$ & Multiple comparison* \\
\hline 1 & $0.68 \pm 0.09$ & $\mathrm{a}$ \\
2 & $0.21 \pm 0.04$ & $\mathrm{~b}$ \\
3 & $0.16 \pm 0.04$ & $\mathrm{~b}$ \\
4 & 0 & $\mathrm{c}$ \\
Cumulative & $1.06 \pm 0.10$ & - \\
\hline
\end{tabular}

* Different letters indicate statistical difference at $\mathrm{P}<0.05$.

In 2018, the cumulative escape rate without tillage (tillage depth of $0 \mathrm{~cm}$ ) was $8.75 \%$ (Table 6 ), which was similar to $7.89 \%$ in 2017 (Table 3). However, it was significantly higher than 
the escape rates of $2.59 \%$ (tillage depth of $20 \mathrm{~cm}$ ) and $2.37 \%$ (tillage depth of $35 \mathrm{~cm}$ ). There was no significant difference between the escape rate of tillage with 20 and $35 \mathrm{~cm}$.

Table 6. Effect of different tillage depth on cumulative escape rate.

\begin{tabular}{lll}
\hline Tillage depth $(\mathbf{c m})$ & $\begin{array}{l}\text { Escape rate (mean } \pm \\
\text { SD }(\%))\end{array}$ & Multiple comparison * \\
\hline 0 & $8.75 \pm 0.78$ & a \\
20 & $2.59 \pm 0.70$ & $\mathrm{~b}$ \\
35 & $2.37 \pm 0.85$ & $\mathrm{~b}$ \\
\hline
\end{tabular}

* Different letters indicate statistical difference at $\mathrm{P}<0.05$.

\section{Discussion}

Overwintering larvae and pupae are an important source of first generation C. suppressalis (Walker) in early spring. Agricultural control of overwintering borers has a long history. In 1971, an unsigned news article reported an example of irrigation in rice fields to kill overwintering pupae of $C$. suppressalis. After irrigation, the mortality of pupae was $43 \%$ on day 1 and the mortality rate reached $78 \%-85 \%$ on day 3 [10]. In the indoor experiment of this study, the mortality rate reached $72 \%-81 \%$ at $72 \mathrm{~h}$ and it was a bit lower (Table 1 ).

Because the pupa itself is unable to move much, immersion usually works. However, there are no published reports on aging larvae. Previous studies have suggested that the tolerance of terrestrial insects to immersion varies. The habitat of Cicindela tuta was often flooded for several days or weeks. The maximum survival time of their larvae was up to $144 \mathrm{~h} \mathrm{(6}$ days) at room temperature, and the $50 \%$ mortality time was $85.9 \mathrm{~h} \mathrm{[12].} \mathrm{Yu} \mathrm{et} \mathrm{al.} \mathrm{(2013)} \mathrm{found} \mathrm{that} \mathrm{the} \mathrm{predicted} \mathrm{50 \%}$ mortality time of minor and major workers of fire ant, Solenopsis invicta, was 16.71 and $19.42 \mathrm{~h}$, respectively, and the total death time was 27.84 and $32.05 \mathrm{~h}$, respectively. Although $C$. suppressalis are terrestrial insects, the larvae live in paddy fields, a high temperature and humid environment. It was easy to speculate that the larvae have strong tolerance to immersion and anoxia. Even so, the results in this study were unexpected, that is, the overwintering $C$. suppressalis larvae survived for as long as 15 days in immersion (and anoxic conditions) (Figure 1). It is possible that some of the overwintering larvae are still in diapause, which raises the question of whether these diapausing larvae are more tolerant to immersion and anoxia. The mechanism of overwintering $C$. suppressalis larvae tolerance to immersion needs further study.

As shown above, the $C$. suppressalis larvae still had great vitality after immersion. Without tillage, the cumulative escape rate over 4 days after immersion was $7.89 \%$ (Table 3 ). If this proportion of the larvae survived, they would become moths and they could aggravate the damage. However, the cumulative escape rate decreased significantly to $1.06 \%$ if tilled immediately after irrigation (Table 5). The combination of irrigation and tillage can greatly reduce the larval escape rate and reduce effective sources.

Since 2010, there have been many migrant workers moving from country to the city in Fenyi County, Jiangxi Province.
The area of double cropping rice has been gradually reduced. The winter fallow fields of single cropping rice have been left untilled even in spring. Thus, the aging larvae and pupae hiding in the fallow fields survived and would probably add to the effective source. This is probably an important cause of the outbreak of C. suppressalis in 2016 [13]. The proposed targeted prevention and control strategy was "Deep tillage and immersion to eliminate pupae (keep deep water for 7-10 days)". This strategy agreed with the results of this study, but the reduction in the large amount of aging larvae might be the most important component. The technical program for the prevention and control of major diseases and insect pests of rice (2017) issued by the National Agricultural Technology Center recommends "filling in rice field with deep water for 7-10 days to reduce the base number of $C$. suppressalis the insect source". The development of overwintering larvae is highly variable and it is difficult for farmers to predict the peak period of pupation in practice.

\section{Conclusion}

The present findings indicated that the aging larvae of $C$. suppressalis have extremely strong tolerance to immersion. The mortality was less than $50 \%$ on day 8 and reached $80 \%$ on day 12 . However, the tolerance of pupa to water was relatively weak. In the field, the cumulative escape rate of larvae was $7.89 \%$ within 4 days after irrigation. But when the plots were tilled immediately after immersion, the cumulative escape rate decreased to $1.05 \%$. "Tilling immediately after irrigation" was recommended in early spring. The results will provide guidance for agricultural control of C. suppressalis.

\section{Acknowledgements}

This study was financed by the National Key Research and Development Plan (2017YFD0301600) with additional finance provided by the Special Fund for Agro-Scientific Research in the Public Interest (201303017) and the National Key Research and Development Plan (2016YFD0200808). The study was also supported by Jiangsu Agriculture Science and Technology Innovation Fund (CX (16) 1001). The authors would like to thank the reviewers for their constructive suggestions. We thank Catherine Dandie, PhD, from Liwen Bianji, Edanz Editing China (www.liwenbianji.cn/ac), for editing the English text of a draft of this manuscript.

\section{References}

[1] Huang, S., et al., Resistance Monitoring of Four Insecticides and a Description of an Artificial Diet Incorporation Method for Chilo suppressalis (Lepidoptera: Crambidae). J Econ Entomol, 2017. 110 (6): p. 2554-2561.

[2] Sun, Y., et al., Chlorantraniliprole resistance and its biochemical and new molecular target mechanisms in laboratory and field strains of Chilo suppressalis (Walker). Pest Manag Sci, 2018.74 (6): p. 1416-1423. 
[3] Gao, X., Management of insecticide resistance. Worldwide Agriculture, 1987 (07): p. 41-44.

[4] Dai, Z., et al., There were significant differences in the effects of different tillage techniques on survival rate of overwintering striped rice (Chilo suppressalis) and yellow stem borer (Tryporyza incertulas). Plant Protection, 1993. 19 (04): p. 48-49.

[5] Xia, S., L. Pingliang, and H. Zhiping, The effect of machine harvesting late rice on numbers of Chilo suppressalis and control countermeasure. Plant Protection Technology and Extension, 2001. 21 (08): p. 10-15.

[6] Wang, Z., C. Yunkang, and P. Guoqiang, Study on control of overwintering Chilo suppressalis population base and damge with restocking ducks in winter fallow fields deep water irrigated. China Plant Protection, 2005. 25 (02): p. 5-6.

[7] Guan, R., et al., Analysis on overwintering characteristics and reason of Chilo suppressalis walker in Fujian. Fujian Agricultural Science and Technology, 2008 (5): p. 49-52.

[8] Wang, X., et al., Plant performance and supporting technology of hybrid rice Yongyou 6 in WenLing. Journal of Zhejiang Agricultural Sciences, 2008 (1): p. 49-51.

[9] Jiang, W., et al., Rice striped stem borer, Chilo suppressalis (Lepidoptera: Pyralidae), overwintering in super rice and its control using cultivation techniques. Crop Protection, 2011. 30 (2): p. 130-133.

[10] Ningbo-Agricultural-Institute, Irrigation to kill pupae is a good method to control Chilo suppressalis Bulletin of science and technology, 1971 (38): p. 13.

[11] Liu, Q., et al., Amelioration Effects of Liming on Acid Soil in Main Rice Producing Areas in Hunan. Hunan Agricultural Sciences, 2014 (13): p. 29-32.

[12] Hoback, W. W., et al., Survival of Immersion and Anoxia by Larval Tiger Beetles, Cicindela togata. The American Midland Naturalist, 1998. 140 (1): p. 27-33.

[13] Gong, Q., Z. Zhenhua, and L. Liang, The Cause of Heavy Occurrence and Prevention and Control Countermeasures of Chilo suppressalis (Walker) in Fenyi County in 2016. Journal of Anhui Agriculture Science, 2017. 45 (26): p. 133-135. 